\title{
BMJ Open Adiposity change and mortality in middle-aged to older Chinese: an 8-year follow-up of the Guangzhou Biobank Cohort Study
}

\author{
Ying Yue Huang, ${ }^{1}$ Chao Qiang Jiang, ${ }^{2}$ Lin Xu (D) , ${ }^{1,3}$ Wei Sen Zhang, ${ }^{2}$ Feng Zhu, ${ }^{2}$ \\ Ya Li Jin, ${ }^{2}$ G Neil Thomas, ${ }^{4}$ Kar Keung Cheng, ${ }^{4}$ Tai Hing Lam ${ }^{2,3}$
}

To cite: Huang YY, Jiang $C Q$, $\mathrm{Xu} \mathrm{L}$, et al. Adiposity change and mortality in middle-aged to older Chinese: an 8-year followup of the Guangzhou Biobank Cohort Study. BMJ Open 2020;10:e039239. doi:10.1136/ bmjopen-2020-039239

- Prepublication history and additional material for this paper is available online. To view these files, please visit the journal online (http://dx.doi.org/10. 1136/bmjopen-2020-039239).

Received 08 April 2020 Revised 05 November 2020 Accepted 16 November 2020

Check for updates

(C) Author(s) (or their employer(s)) 2020. Re-use permitted under CC BY-NC. No commercial re-use. See rights and permissions. Published by BMJ.

${ }^{1}$ School of Public Health, Sun Yat-sen University, Guangzhou, China

${ }^{2}$ Molecular Epidemiology Research Centre, Guangzhou No.12 Hospital, Guangzhou, China

${ }^{3}$ School of Public Health, the University of Hong Kong, Hong Kong, China

${ }^{4}$ Institute of Applied Health Research, University of Birmingham, Birmingham, UK

\section{Correspondence to}

Professor Lin Xu;

xulin27@mail.sysu.edu.cn and

Dr Chao Qiang Jiang;

cqjiang@hku.hk

\section{ABSTRACT}

Objective To examine the associations of change in body mass index (BMI) and waist circumference (WC) over an average of 4 years with subsequent mortality risk in middle-aged to older Chinese.

Design Prospective cohort study based on the Guangzhou Biobank Cohort Study.

Setting Community-based sample.

Participants 17773 participants (12 956 women and 4817 men) aged $50+$ years.

Primary and secondary outcome measures Primary outcome measure was all-cause mortality. Secondary outcome measures were cardiovascular disease (CVD) and cancer mortality. Causes of death were obtained via record linkage, and coded according to the International Classification of Diseases (tenth revision).

Results 1424 deaths ( $53.4 \%$ women) occurred in the 17 773 participants (mean age 61.2, SD 6.8 years) during an average follow-up of $7.8(\mathrm{SD}=1.5)$ years, and $97.7 \%$ of participants did not have an intention of weight loss . Compared with participants with stable BMI, participants with BMl loss $(>5 \%)$, but not gain, had a higher risk of all-cause mortality ( $\mathrm{HR}=1.49,95 \% \mathrm{Cl} 1.31$ to 1.71 ), which was greatest in those who were underweight ( $\mathrm{HR}=2.45$, $95 \% \mathrm{Cl} 1.31$ to 4.59 ). Similar patterns were found for WC. In contrast, for participants with a BMl of $\geq 27.5 \mathrm{~kg} / \mathrm{m}^{2}$, BMI gain, versus stable BMl, was associated with $89 \%$ higher risk of all-cause mortality ( $\mathrm{HR}=1.89,95 \% \mathrm{Cl} 1.25$ to 2.88 ), $72 \%$ higher risk of CVD mortality $(\mathrm{HR}=1.72,95 \% \mathrm{Cl} 0.80$ to 3.72 ) and 2.27-fold risk of cancer mortality ( $\mathrm{HR}=2.27$, $95 \% \mathrm{Cl} 1.26$ to 4.10$)$.

Conclusion In older people, unintentional BMI/WC loss, especially in those who were underweight was associated with higher mortality risk. However, BMl gain in those with obesity showed excess risks of all-cause and cancer mortality, but not CVD mortality. Frequent monitoring of changes in body size can be used as an early warning for timely clinical investigations and interventions and is important to inform appropriate health management in older Chinese.

\section{INTRODUCTION}

Obesity is a rapidly expanding public health problem worldwide. The prevalence of overweight and obesity has been increasing sharply
Strengths and limitations of this study

- This is a large population-based longitudinal study incorporating adiposity assessed at two time points and comprehensive measurements of potential confounders.

- The relatively short follow-up period might lead to insufficient number of deaths and thus a null association.

- Due to the lack of sufficient data on intentional weight loss, the association of intentional weight loss and mortality in our study could not be examined.

- Due to the observational nature of the study, biases from survival effects and residual confounding could not be ruled out, and hence the observed associations should not be interpreted causally.

- Generalisability to younger populations might be limited because all participants were older Chinese.

in most countries over the past few decades. ${ }^{1}$ A four stages of obesity epidemic model proposed that in most countries obesity is in the early stages (stages 1 and 2) with increasing prevalence. ${ }^{2}$ China is now in stage 1 , with a relatively low $(<10 \%)$ prevalence of obesity, and the morbidity and mortality outcomes attributed to obesity are not yet evident. ${ }^{2}$ Previous studies have shown that individuals with obesity at baseline had higher risks of mortality during follow-up. ${ }^{3}$ However, the relative mortality risk associated with obesity at baseline only might be biassed because body weight might have changed before baseline and/or during follow-up. Such issues had been highlighted by the controversy on whether overweight was protective or harmful in terms of its association with all-cause mortality. ${ }^{34}$ Assessment of weight fluctuation at multiple time points might partly clarify these problems. Two recent systematic reviews of prospective cohort studies with data at two time points showed that both weight loss and 
weight gain from baseline were associated with higher risks of all-cause mortality. ${ }^{56}$ Most of the studies included in these two reviews were from high-income countries in Europe and North America, and only five studies were from Asia published before $2015 .^{7-11}$ Meanwhile, due to the lack of information on lifestyle, health and weight loss intention, residual confounding might be substantial, and reverse causality might counteract or overwhelm the associations of weight changes with mortality risk. ${ }^{12}$ Moreover, baseline obesity might have modified the associations of subsequent adiposity change with the risks of mortality, ${ }^{13} 14$ highlighting the importance of stratifying by baseline obesity status.

Rapid economic growth is often followed by increasing adiposity which is compounded by the ageing of the population. The prevalence of obesity in China has been increasing since the open-door policy in 1978. However, we found only two cohort studies from China examining the mortality risk due to adiposity changes. Both cohorts were small ( $\mathrm{n}=1696$ or 2090), and body mass index (BMI) changes during the early economic development (19761994 or 1992-2000) could not represent adiposity changes over recent years, ${ }^{1015}$ as the prevalence of overweight and obesity has been increasing dramatically together with ageing in China since 2000. ${ }^{16}$ Moreover, using BMI alone could not distinguish body fat distribution and may lead to misclassification of adiposity.

Hence, in the present study, we used data from the Guangzhou Biobank Cohort Study (GBCS) to examine the associations of BMI and waist circumference (WC) change over an average of 4 years with subsequent mortality risk. We hypothesised that in older people, $\mathrm{BMI} / \mathrm{WC}$ loss would be associated with higher risks of mortality, while BMI/WC gain, indicating real adiposity increase, in the obese group would be associated with higher mortality risks.

\section{METHODS}

\section{Study subjects}

At baseline of GBCS, 30430 participants aged 50 years or above were recruited from 2003 to 2008. All surviving participants were invited for a repeated physical examination from March 2008 to December 2012 (ie, the first follow-up). Details of baseline examination and some results from the first follow-up examination have been reported previously. ${ }^{17-20}$ Briefly, the GBCS is a three-way collaboration among the Guangzhou 12th Hospital and the Universities of Hong Kong and Birmingham. Participants were recruited from 'The Guangzhou Health and Happiness Association for the Respectable Elders' (GHHARE), a community social and welfare organisation. GHHARE is unofficially aligned with the municipal government. Membership is open to permanent Guangzhou residents aged 50 years or above for a nominal fee of $¥ 4$ ( 50 US cents) per month. GHHARE includes about $7 \%$ of Guangzhou residents in this age group, and has branches in all 10 districts of Guangzhou. The baseline and the first follow-up examinations included a face-toface interview by trained nurses using a computer-assisted standardised questionnaire that included demographic characteristics, lifestyle factors, family and personal medical history, and assessment of height, weight, WC, blood pressure, fasting plasma glucose and lipids, and inflammatory markers. Weight loss intention was ascertained by the question 'have you tried to reduce your weight in the past 12 months?'. The first follow-up examinations did not include height. We assumed that the height of participants did not change within the past few years. All participants gave written, informed consent before participation.

\section{Patient and public involvement}

No patients or public were involved in setting the research question or the outcome measures, nor were they involved in the design and implementation of the study. There are no plans to involve patients in dissemination.

\section{Measures of adiposity change}

Anthropometric measurements were performed by trained nurses using standard protocols in the morning before breakfast, including weight, standing height and WC, with light clothing and no shoes. WC was measured horizontally around the smallest circumference between the ribs and iliac crest, or at the navel, if no natural waistline was present. BMI was calculated as weight divided by height squared $\left(\mathrm{kg} / \mathrm{m}^{2}\right)$. As the body build varies by ethnicity, we used the Chinese-specific cut-offs recommended by the WHO. Underweight was defined as BMI lower than $18.5 \mathrm{~kg} / \mathrm{m}^{2}$, normal weight as BMI $18.5-24.9 \mathrm{~kg} / \mathrm{m}^{2}$, overweight as BMI $25.0-27.4 \mathrm{~kg} / \mathrm{m}^{2}$ and obesity as BMI $\geq 27.5 \mathrm{~kg} / \mathrm{m}^{2}{ }^{21}$ Central adiposity was defined as $W C \geq 90 \mathrm{~cm}$ in men and $\geq 80 \mathrm{~cm}$ in women. ${ }^{22}$

BMIs measured at baseline $\left(\mathrm{BMI}_{1}\right)$ and at the first follow-up examination $\left(\mathrm{BMI}_{2}\right)$ were used to calculate the proportional change for each individual: $\Delta \mathrm{BMI}=\left(\left(\mathrm{BMI}_{2}-\right.\right.$ $\left.\left.\mathrm{BMI}_{1}\right) / \mathrm{BMI}_{1}\right) * 100 \%$. As definitions of substantial weight change ranged from $3 \%$ to $5 \%$ in previous studies (for example, a stable weight was defined as weight changes within $\pm 3 \%$ by Lee $e t a l^{23}$ and within $\pm 5 \%$ by Pan $e t a l^{24}$ and Dahl et $a l^{25}$ ), we chose a relatively conservative definition (ie, $5 \%$ as the cut-off) for BMI change. Participants were classified into three groups based on their proportional BMI change: BMI loss $(\Delta \mathrm{BMI}<-5 \%)$, BMI stable $(-5 \%$ to $5 \%)$, and $\mathrm{BMI}$ gain $(\triangle \mathrm{BMI}>5 \%)$. The same classification method was used for WC change. ${ }^{11}$

\section{Mortality}

Information on underlying causes of deaths up to December 2017 was mostly obtained via record linkage with the Death Registry Department of the Guangzhou Centre for Disease Control and Prevention. The methods have been described in detail elsewhere. ${ }^{26}{ }^{27}$ Briefly, causes of death were coded according to the International Classification of Diseases (tenth revision) by trained medical staff in each hospital. In addition, verbal 
30430 participants from 2003-2008

- Phase $1 \mathrm{~N}=10381$ (2003-4)

- Phase $2 \mathrm{~N}=10000$ (2005-6)

- Phase $3 \mathrm{~N}=10049$ (2006-8)

Excluding participants with:

- loss to follow-up with unknown vital status $(\mathrm{n}=384)$

- missing information on the $1^{\text {st }}$ repeated examination $(\mathrm{n}=12$ 326)

- incomplete information on body mass index (BMI) and waist circumference (WC) $(\mathrm{n}=282)$

- BMI loss/gain $>50 \%(n=49)$

Figure 1 Flow diagram.

autopsy meetings were conducted in the Guangzhou 12th Hospital to further clarify the deaths with unclear causes. In the present study, we analysed all-cause mortality, and mortality due to all cardiovascular diseases (CVDs) and all cancers.

\section{Statistical analysis}

The Pearson $\chi^{2}$ tests and one-way analysis of variance were used to compare baseline characteristics of groups according to $\mathrm{BMI} / \mathrm{WC}$ change. Cox regression was used to analyse associations of mortality with $\mathrm{BMI} / \mathrm{WC}$ change, giving crude and adjusted HRs and 95\% CIs. Potential confounders included sex, age (continuous), education (primary, middle school and college), occupation (manual, non-manual and others), personal income

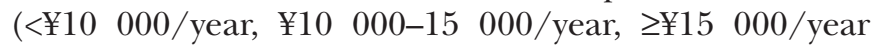
and not known, US\$1 $\approx ¥ 8)$, physical activity assessed by International Physical Activity Questionnaire (inactive, moderate and active), ${ }^{28}$ alcohol drinking (never, former and current drinkers), smoking (never, former and current smokers) and self-rated health (very good, good, poor and very poor). The study period used in the calculation of person years started from the first follow-up examination. To alleviate the effect of the time interval between the two examinations, we conducted subgroup analysis by time interval between the baseline and follow-up measurements ( $<4$ years $/ \geq 4$ years). To limit biases from underlying illnesses, analyses were also done after excluding participants who had poor health status at baseline and deaths within the first 3 years. Poor health status at baseline was defined by the presence of any of the following: (1) Regular use of medications for chronic diseases, such as diabetes, hypercholesterolaemia or CVDs, or (2) Any hospital admission in the past 6 months before baseline examination, or (3) Self-reported CVD history, or (4) Self-reported cancer history. ${ }^{29}$ We also evaluated if the associations varied by age, baseline obesity status or weight loss intention between two examinations. Moreover, to avoid the issue of setting arbitrary cut-offs and increase statistical power, we also analysed associations of mortality with proportional BMI/WC change as continuous variables. Participants who died of any other causes after the first follow-up examination were regarded as censored at the date of death. ${ }^{30}$ All analyses were done using STATA/IC V.14.0 (StataCorp LP, CollegeStation, Texas, USA). All p values were two-sided, and statistical significance was defined as $\mathrm{p}<0.05$.

\section{RESULTS}

Of the 30430 participants at baseline, 12657 were excluded, and of them, 384 were lost to follow-up with unknown vital status, 12326 did not return for the first repeated examination, 282 had incomplete information on BMI or WC, and 49 had BMI loss/gain $>50 \%$ (in this cohort, as mean BMI increased only slightly, ${ }^{29}$ change $>50 \%$ was over the 99th centile and might be due to measurement errors), giving 17773 participants (12 956 women and 4817 men) in the present analysis (figure 1$)$. The mean age was $61.2(\mathrm{SD}=6.8)$ years. During the average follow-up of $7.8(\mathrm{SD}=1.5)$ years since the first follow-up examination in 2008-2012 to December 2017, 1424 (761 (53.4\%) women and $663(46.6 \%)$ men) deaths were recorded.

Table 1 shows that during baseline and the first follow-up examination, most of the participants $(68.4 \%)$ had stable BMI with $\pm 5 \%$ change; $13.7 \%$ had BMI loss of $>5 \%$ and $17.9 \%$ had BMI gain of $>5 \%$. For WC, more participants $(50.0 \%)$ had a gain of $>5 \%$ than having stable WC within $\pm 5 \%$ change $(42.6 \%)$, and few $(7.4 \%)$ had WC loss of $>5 \%$. At baseline, compared with those with stable $\mathrm{BMI}$, those with BMI loss were older, more likely to be women, with poor health status, intentional weight loss and type 2 diabetes ( $p$ from $<0.001$ to 0.024 ), and with lower socioeconomic position indicated by low education, manual occupation and low family income ( $\mathrm{p}$ from $<0.001$ to 0.032 ). Those with BMI gain were younger, more likely to be women, manual workers and with good health status ( $\mathrm{p}$ from $<0.001$ to 0.001 ), and less likely to be with intentional weight loss and type 2 diabetes ( $p$ from $<0.001$ to 0.024 ). Those with BMI loss or BMI gain were less likely to be physically active and alcohol users than those with stable BMI ( $p$ from <0.001 to 0.004). Even though statistically significant, the differences were small. Baseline characteristics by WC change showed similar patterns (table 1). Among those with BMI gain, 
Table 1 Baseline characteristics of 17773 participants aged 50+ years in the Guangzhou Biobank Cohort Study first examined in 2003-2008 and followed up until December 2017

\begin{tabular}{|c|c|c|c|c|}
\hline & \multicolumn{3}{|l|}{ BMI change } & \multirow[b]{2}{*}{$P$ value for difference } \\
\hline & Loss $(<-5 \%)$ & Stable $(-5 \%$ to $5 \%)$ & Gain (>5\%) & \\
\hline Number of participants (n, \%) & 2435 (13.7) & $12165(68.4)$ & 3173 (17.9) & \\
\hline Age, years, mean (SD) & $62.2(7.3)$ & $61.1(6.8)$ & $60.8(6.6)$ & $<0.001$ \\
\hline Sex, \% men & 26.5 & 28.0 & 24.1 & $<0.001$ \\
\hline Education, \% primary or below & 39.0 & 38.7 & 40.6 & $<0.001$ \\
\hline Occupation, \% manual & 60.8 & 59.8 & 61.4 & $<0.001$ \\
\hline Family income ( $¥ /$ year), $\%<30000$ & 38.9 & 36.3 & 37.9 & 0.032 \\
\hline Personal income ( $¥ /$ year), $\%<10000$ & 31.2 & 31.2 & 35.2 & $<0.001$ \\
\hline Physical activity, \% active & 51.8 & 52.4 & 51.7 & 0.004 \\
\hline Alcohol use, \% current & 23.2 & 26.2 & 23.1 & $<0.001$ \\
\hline Smoking, \% current & 9.0 & 9.3 & 10.0 & 0.199 \\
\hline Self-rated health, \% poor & 16.9 & 16.2 & 18.5 & 0.012 \\
\hline Health status, \% poor & 21.0 & 18.7 & 17.2 & 0.001 \\
\hline Intentional weight loss, ${ }^{*} \%$ & 3.0 & 2.3 & 1.9 & 0.024 \\
\hline \multirow[t]{3}{*}{ Type 2 diabetes, \% yes } & 7.8 & 5.5 & 5.4 & $<0.001$ \\
\hline & \multicolumn{4}{|c|}{ Waist circumference change } \\
\hline & Loss $(<-5 \%)$ & Stable $(-5 \%$ to $5 \%)$ & Gain $(>5 \%)$ & $P$ value for difference \\
\hline Number of participants (n, \%) & $1310(7.4)$ & $7573(42.6)$ & $8890(50.0)$ & \\
\hline Age, years, mean (SD) & $62.9(6.7)$ & $61.7(6.7)$ & $60.5(6.9)$ & $<0.001$ \\
\hline Sex, \% men & 31.2 & 32.0 & 22.2 & $<0.001$ \\
\hline Education, \% primary or below & 39.5 & 39.3 & 38.8 & $<0.001$ \\
\hline Occupation, \% manual & 58.1 & 60.4 & 60.3 & $<0.001$ \\
\hline Family income ( $¥ /$ year), $\%<30000$ & 41.3 & 36.3 & 36.8 & $<0.001$ \\
\hline Personal income ( $¥ /$ year), $\%<10000$ & 32.4 & 31.2 & 32.4 & $<0.001$ \\
\hline Physical activity, \% active & 56.5 & 55.0 & 49.1 & $<0.001$ \\
\hline Alcohol use, \% current & 22.9 & 25.6 & 25.3 & 0.001 \\
\hline Smoking, \% current & 10.8 & 10.6 & 8.0 & $<0.001$ \\
\hline Self-rated health, \% poor & 17.2 & 16.3 & 17.0 & 0.480 \\
\hline Health status, \% poor & 21.2 & 20.1 & 17.2 & $<0.001$ \\
\hline Intentional weight loss, ${ }^{\star} \%$ & 3.4 & 2.6 & 2.0 & 0.001 \\
\hline Type 2 diabetes, \% yes & 8.0 & 6.8 & 4.6 & $<0.001$ \\
\hline
\end{tabular}

$¥ \approx 12.5$ US cents, US $\$ 1 \approx ¥ 8$

*Based on weight loss intention between two examinations

BMI, body mass index.

the prevalence of underweight $(7.06 \%)$ at baseline was higher than those with stable BMI or BMI loss (prevalence $3.58 \%$ and $2.87 \%$, respectively). A similar pattern was seen among WC change groups. However, those with BMI loss had higher prevalence of overweight (23.33\%) or obesity (15.93\%) at baseline than those with stable BMI (22.22\% and $12.91 \%$, respectively) or BMI gain $(15.73 \%$ and $8.07 \%$, respectively) (see online supplemental table $1)$.

\section{BMI change and mortality}

Table 2 shows that after adjusting for sex, age, education, occupation, personal income, physical activity, drinking, smoking and self-rated health, compared with participants with stable BMI, those with BMI loss showed a $49 \%$ higher risk for all-cause mortality $(\mathrm{HR}=1.49,95 \%$ CI 1.31 to 1.71$), 45 \%$ higher risk for CVD mortality $(\mathrm{HR}=1.45$, 95\% CI 1.16 to 1.81 ) and $40 \%$ higher risk for cancer mortality (HR=1.40, 95\% CI 1.13 to 1.74 ). No significant association with all-cause mortality risk was found for BMI gain (HR $=0.99$, 95\% CI 0.84 to 1.05$)$. The results were similar after excluding those with poor health at baseline. BMI loss, but not BMI gain, showed higher risks for allcause, CVD and cancer mortality (HR $=1.58,95 \%$ CI 1.36 to $1.85 ; 1.54,95 \%$ CI 1.17 to 2.02 , and $1.57,95 \%$ CI 1.23 


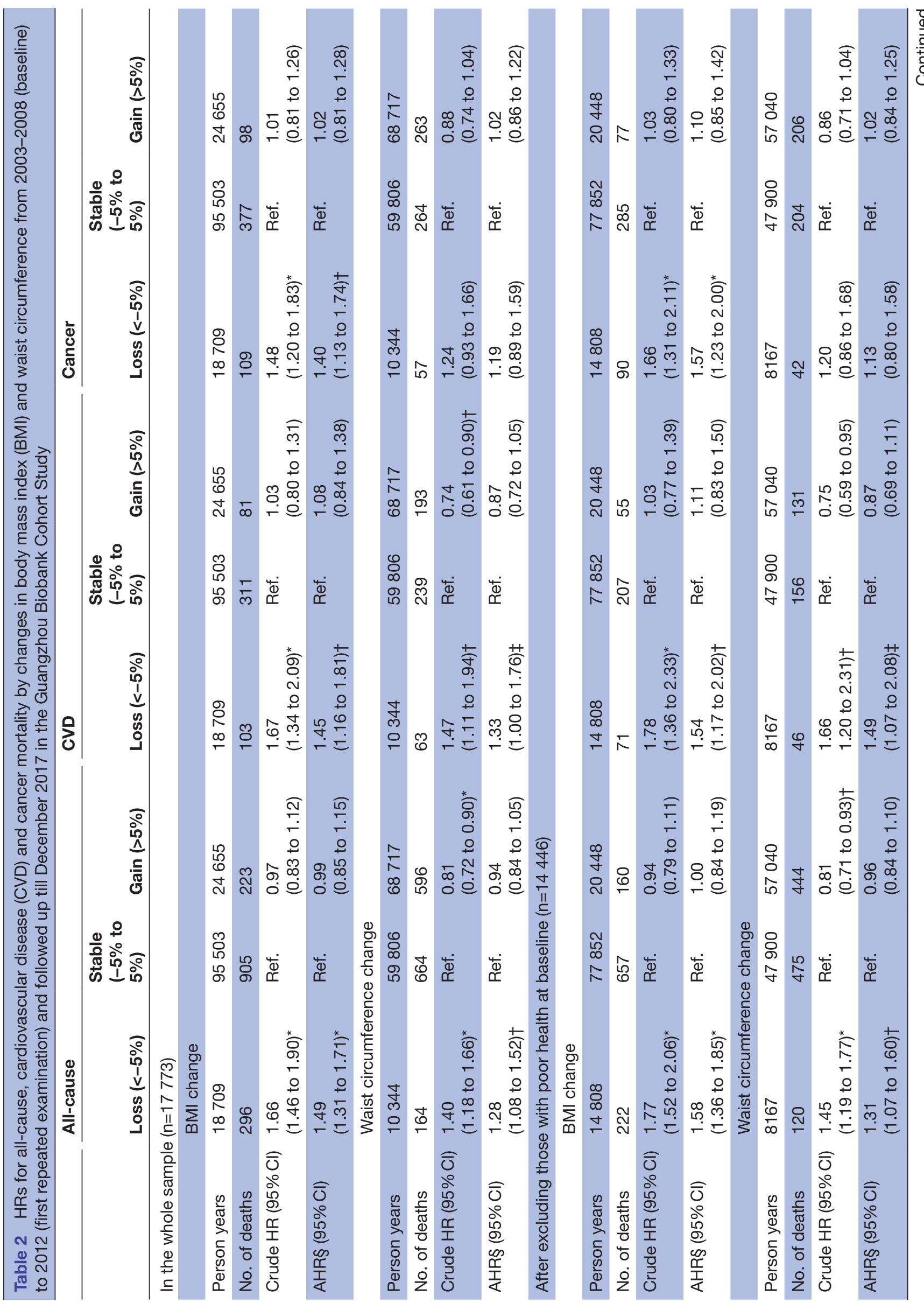

\begin{tabular}{l} 
W \\
3 \\
2 \\
0 \\
0 \\
0 \\
0 \\
\hline
\end{tabular} 


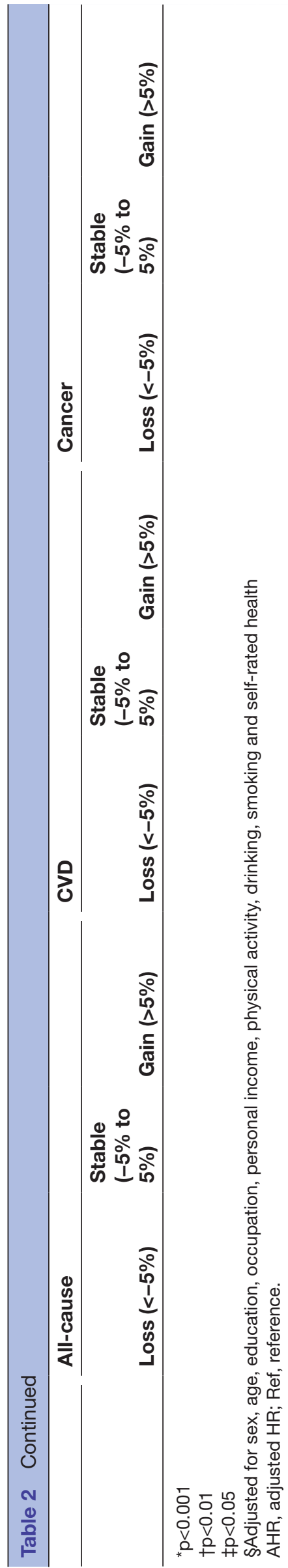

to 2.00 , respectively). The results did not vary substantially after excluding deaths within the first 3 years, or those with intentional weight loss, or stratifying by age at baseline or time interval ( $<4$ years $/ \geq 4$ years) (see online supplemental tables 2-6). However, the HR for mortality risk due to BMI loss was slightly lower after excluding deaths within the first 3 years and in participants aged under 70 years (see online supplemental tables 2and 3). Moreover, when BMI change was used as a continuous variable, for those with BMI loss, less BMI change showed lower mortality risks ( $\mathrm{HR}=0.97,95 \%$ CI 0.96 to 0.99 , per $1 \%$ change in $\mathrm{BMI}$ ), indicating that greater BMI loss was associated with higher mortality risks, while for those with BMI gain, no association with mortality risks was found for BMI change ( $\mathrm{HR}=1.01,95 \% \mathrm{CI} 0.99$ to 1.03 , per $1 \%$ change in BMI) (see online supplemental table 7).

Stratified analyses by baseline BMI status showed different patterns of mortality risk associated with BMI change. Table 3 and figure 2 show that in all four groups with different baseline BMI status, compared with stable BMI, BMI loss showed higher risks for all-cause mortality. In the underweight group, those with BMI loss, versus those with stable BMI, had a high risk for all-cause mortality (HR=2.45, 95\% CI 1.31 to 4.59 ). Compared with the underweight group, the HRs for all-cause mortality in the normal weight, overweight and obese groups were attenuated and became non-significant in the obese group (HR and 95\% CI 1.46 (1.23 to 1.74), 1.52 (1.15 to 2.02 ) and 1.41 (0.98 to 2.02), respectively).

Table 3 and figure 2 also show that in those with obesity at baseline, BMI gain versus stable BMI showed $82 \%$ higher risk of all-cause mortality ( $\mathrm{HR}=1.89,95 \%$ CI 1.25 to 2.88) and more than twofold risk of cancer mortality $(\mathrm{HR}=2.27,95 \%$ CI 1.26 to 4.10$)$. However, when baseline adiposity, ignoring adiposity change, was analysed, the associations were weak and non-significant, with the HR (95\% CI) being 1.17 (0.99 to 1.37) for all-cause mortality and 1.17 (0.91 to 1.51) for cancer mortality (see online supplemental table 8 ). Similar results were observed after excluding those with poor health at baseline (see online supplemental table 9).

\section{WC change and mortality}

Table 2 shows that after adjusting for potential confounding factors as above, WC loss versus WC stable group showed higher risks for all-cause $(\mathrm{HR}=1.28,95 \% \mathrm{CI}$ 1.08 to 1.52$)$ and CVD (HR=1.33, 95\% CI 1.00 to 1.76 ) mortality. No significant associations with mortality risks $(\mathrm{HR}=0.94,95 \%$ CI 0.84 to 1.05$)$ were found for WC gain. The results were similar after excluding those with poor health at baseline, deaths within the first 3 years or intentional weight loss, or stratifying by age at baseline or time interval ( $<4$ years $/ \geq 4$ years) (table 2 , online supplemental tables 2-6). Moreover, when WC change was used as a continuous variable, for those with WC loss, less WC change appeared to be associated with a lower risk of all-cause mortality, although the association was 


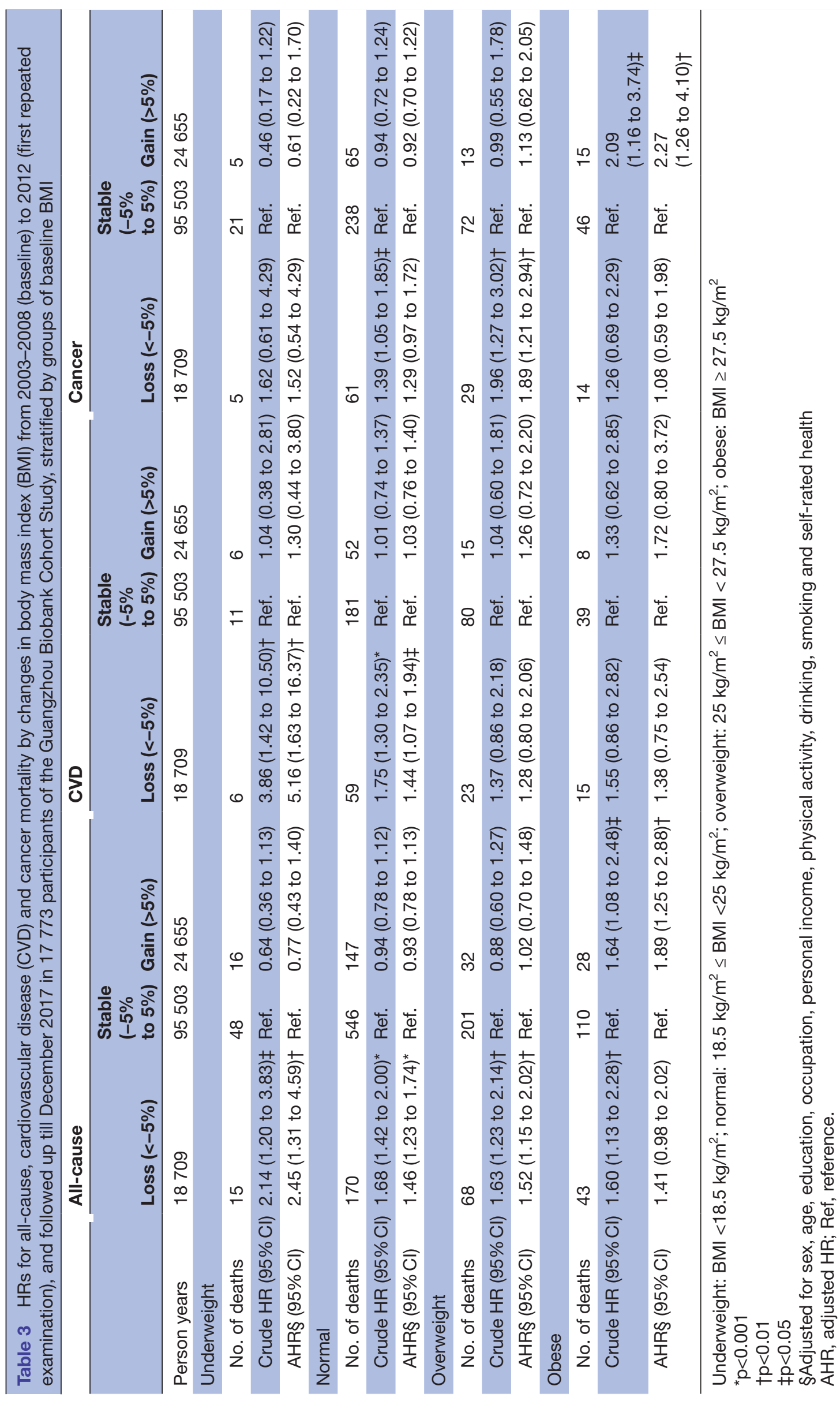




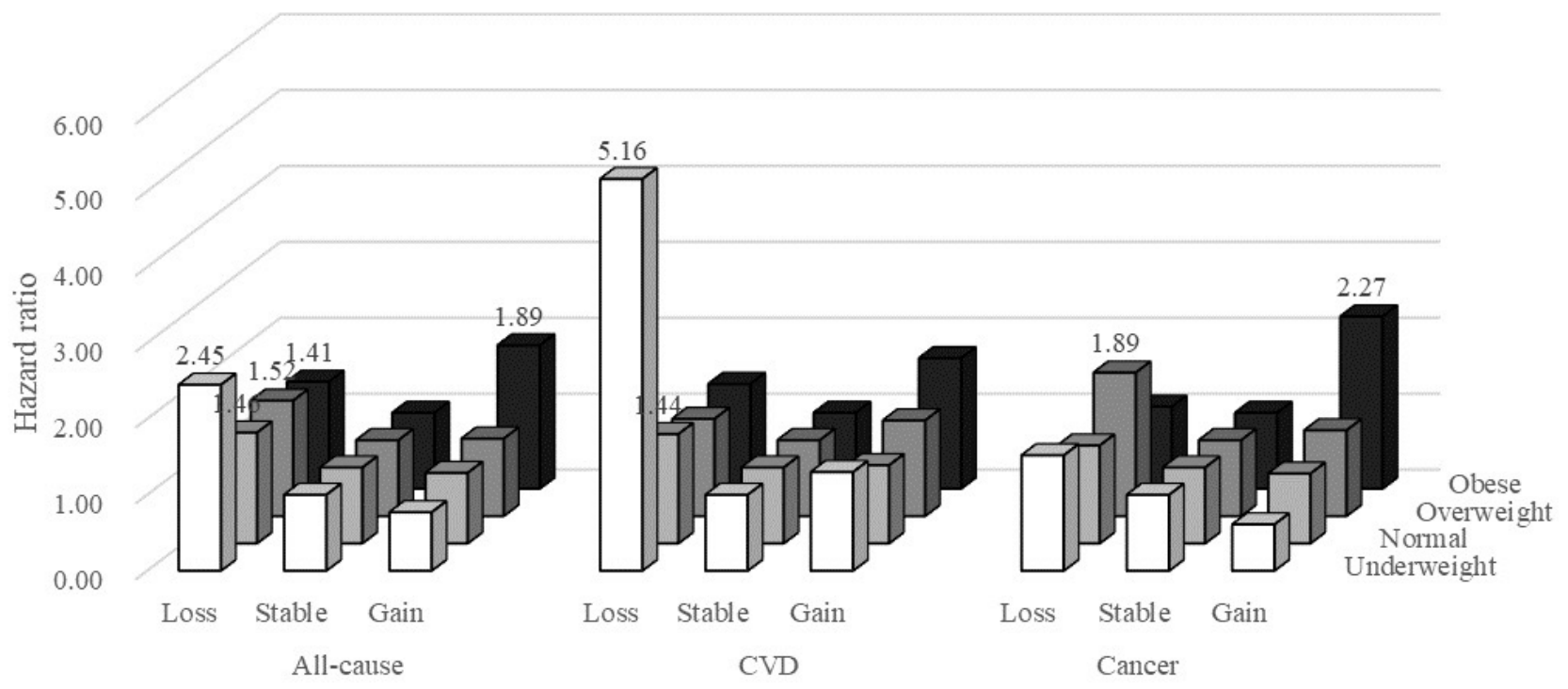

Figure 2 Adjusted HRs* for all-cause, cardiovascular disease (CVD) and cancer mortality by changes in body mass index (BMI) from 2003-2008 (baseline) to 2012 (first repeated examination), and followed up till December 2017 in 17773 participants of the Guangzhou Biobank Cohort Study, stratified by groups of baseline BMl. *: adjusted for sex, age, education, occupation, personal income, physical activity, drinking, smoking and self-rated health. Underweight: BMI $<18.5 \mathrm{~kg} / \mathrm{m}^{2} ; \mathrm{normal:} 18.5 \mathrm{~kg} / \mathrm{m}^{2} \leq$ $\mathrm{BMI}<25 \mathrm{~kg} / \mathrm{m}^{2}$; overweight: $25 \mathrm{~kg} / \mathrm{m}^{2} \leq \mathrm{BMl}<27.5 \mathrm{~kg} / \mathrm{m}^{2}$; obese: $\mathrm{BMI} \geq 27.5 \mathrm{~kg} / \mathrm{m}^{2}$.

not statistically significant $(\mathrm{HR}=0.98,95 \%$ CI 0.96 to 1.00 , per $1 \%$ change in WC) (see online supplemental table 7 ).

Table 4 and figure 3 show that, in men with WC level of $80-90 \mathrm{~cm}$ and women with WC of $70-80 \mathrm{~cm}$, those with WC loss had higher risks for all-cause mortality $(\mathrm{HR}=1.61$, $95 \%$ CI 1.21 to 2.13$)$ and CVD mortality ( $\mathrm{HR}=1.76$, $95 \%$ CI 1.12 to 2.79 ). No significant associations between WC gain and mortality risks in all subgroups were found with HR (95\% CI) being 0.87 (0.69 to 1.10$), 1.05$ (0.87 to 1.27 ) and 0.97 (0.79 to 1.18 ) (table 4 , online supplemental table 10).

\section{DISCUSSION}

To our knowledge, the current study is the largest population-based prospective study in China examining the association of adiposity change with the risks of mortality in middle-aged and older people. The findings in our study are similar to those in the western populations. We found that in participants who were obese (BMI $\geq 27.5 \mathrm{~kg} / \mathrm{m}^{2}$ ) at baseline, BMI gain during follow-up was associated with a higher risk of all-cause and cancer mortality, but not CVD mortality, although the null association for CVD mortality could be due to the lack of statistical power. Notably, when adiposity change was ignored, no significant associations of baseline obesity with the risks of all-cause and cause-specific mortality were observed.
$\mathrm{BMI} / \mathrm{WC}$ loss versus stable BMI showed increased risks of all-cause, CVD and cancer mortality. Moreover, the risks for all-cause and CVD mortality related to BMI loss appeared to be the highest in those who were underweight, followed by those with normal or overweight. Such results which have not been reported before, indicate that reverse causality effects might be likely, and such effects could not be completely eliminated after adjusting for self-rated health and excluding those with poor health at baseline.

Our study's positive associations of BMI loss with allcause, CVD and cancer mortality, and WC loss with allcause mortality are generally consistent with those from two meta-analyses of studies mainly from the West. ${ }^{56}$ The findings could be explained by the reduction of lean body mass with ageing and adiposity loss, which could increase the risk of sarcopenia and frailty. ${ }^{32} 33$ Another possible explanation is due to reverse causality, because adiposity loss in older people is usually indicative of underlying diseases. In our commentary on the more recent meta-analysis on adiposity change, ${ }^{12}$ we highlight that the association of adiposity loss with higher risks of all-cause mortality in middle-aged to older people might be most likely due to reverse causality. ${ }^{12}$ To address reverse causality, the two meta-analyses above conducted subgroup analyses by baseline age and 


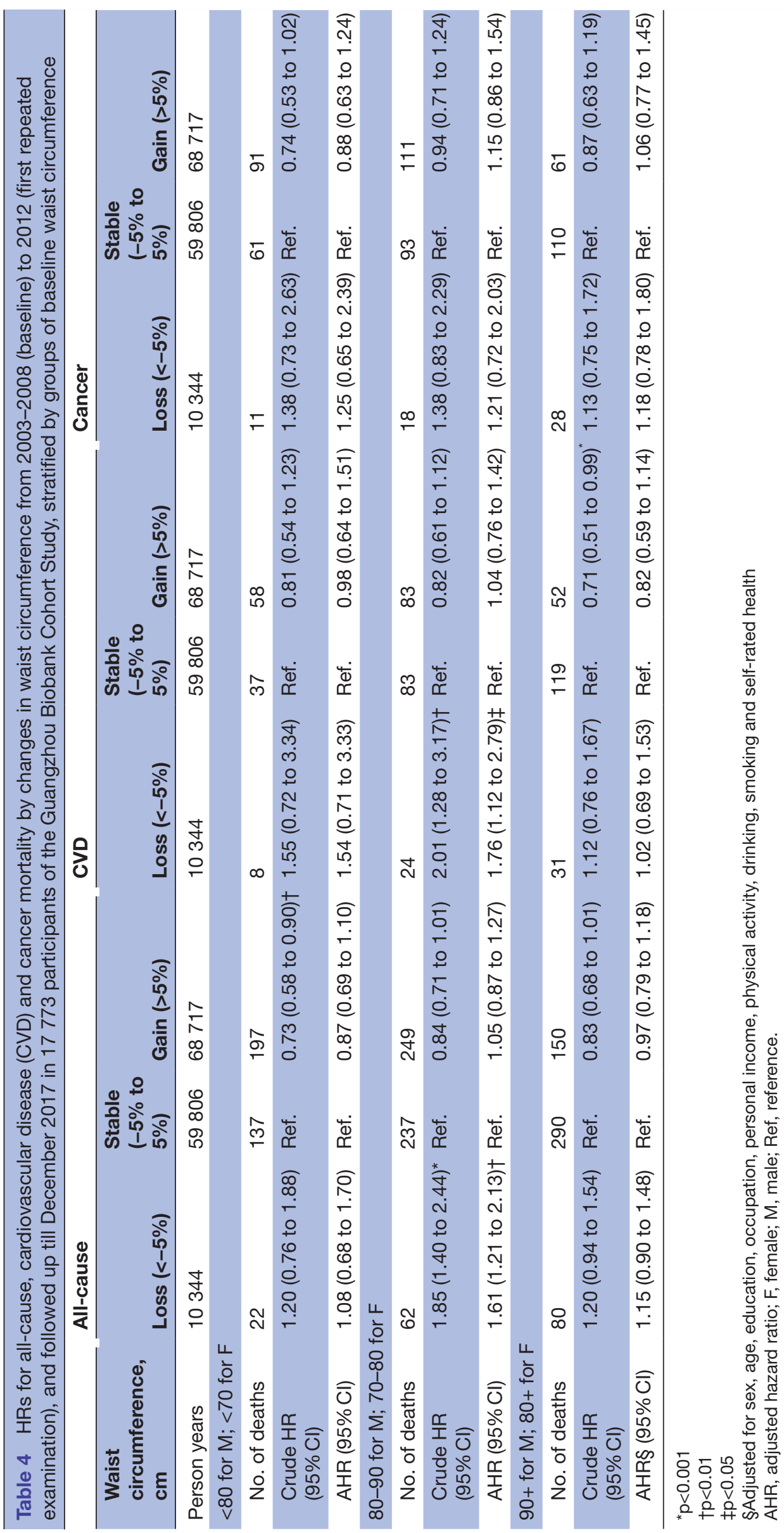




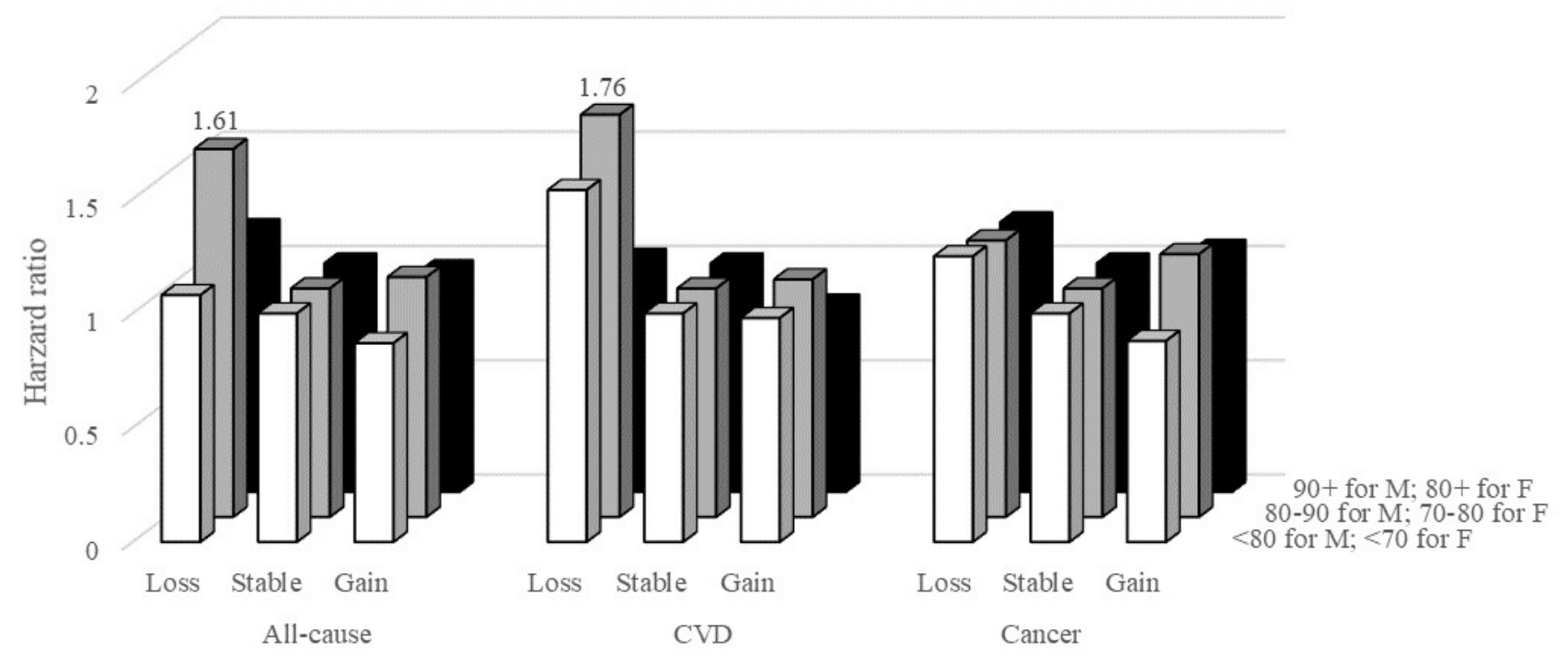

Figure 3 Adjusted $\mathrm{HRs}^{*}$ for all-cause, cardiovascular disease (CVD) and cancer mortality by changes in waist circumference from 2003-2008 (baseline) to 2012 (first repeated examination), and followed up till December 2017 in 17773 participants of the Guangzhou Biobank Cohort Study, stratified by groups of baseline waist circumference (cm). F, female; M, male. *: adjusted for sex, age, education, occupation, personal income, physical activity, drinking, smoking and self-rated health.

follow-up duration, and sensitivity analyses by excluding studies that included potentially unhealthy participants and examining intentional weight loss. They found that the results did not change substantially. The positive associations above were also reported by some recent Asian cohort studies (in China ${ }^{15}$ Singapore ${ }^{24}$ and $\operatorname{Japan}^{9}$ ). In the present study, to explore potential reverse causality, we excluded participants with poor health at baseline or intentional weight loss between two examinations and deaths during the first 3 years, conducted subgroup analyses by age groups ( $<70$ years or $\geq 70$ years), time interval $(<4$ years $/ \geq 4$ years $)$ and baseline BMI status, and found that the results did not vary substantially. Our subgroup analysis showed that the old and thin (underweight) participants had much higher risks of all-cause mortality, which could be an early indicator of frailty and would be of great clinical significance. Our results were consistent with previous studies. ${ }^{13} 15$ However, as expected, the risk of all-cause mortality associated with BMI loss attenuated after excluding deaths within the first 3 years, and the risk in participants aged over 70 years was slightly higher than those aged under 70 years, implying strong reverse causality. As the HRs were the highest in those who were underweight, and almost all weight loss was unintentional, the results should have important clinical applications. Older and underweight people with unintentional BMI loss are at very high mortality risk and need urgent clinical interventions and treatments. For prevention and early detection, the weight of 'old and thin' people should be monitored frequently.

Previous findings on weight gain and mortality were inconsistent, with some studies showing positive associations, ${ }^{9} 1015242534-36$ while others found no association. ${ }^{37-41}$ Our results of the higher risks of all-cause and cancer mortality in those with baseline obesity were consistent with some previous studies, ${ }^{13} 14$ indicating modifying effect of baseline adiposity status. The associations might be explained by the increase in excess body fat and adiposity tissue, which lead to higher inflammation, alteration of immune response and subsequent risks for metabolic disorders. ${ }^{42} 43$ The two meta-analyses above, without weight change data, showed consistently that obesity at baseline was associated with higher risks of all-cause mortality during follow-up. ${ }^{34}$ Thus, BMI gain from baseline obesity would result in higher mortality risks. However, if participants with underweight atbaseline gained BMI, as a result of improved nutrition and health status from treatment, their BMI might approach a normal level, and the benefitof normal BMI level might counteract the adverse effect of BMI gain. Note that obesity as defined in our sample has a low BMI $\left(\geq 27.5 \mathrm{~kg} / \mathrm{m}^{2}\right)$ than the WHO definition $\left(\geq 30.0 \mathrm{~kg} / \mathrm{m}^{2}\right)$, and gross obesity is rare in China and was rare in previous meta-analyses. With increasing obesity and ageing in China and globally, in prevalence and in absolute BMI levels, future studies 
would probably show greater relative risks of mortality and disease burdens due to adiposity.

The strengths of our study included the populationbased longitudinal design incorporating adiposity measured at two time points, comprehensive measurements and analyses of a wide range of potential confounding factors, and large sample size. In addition, information of anthropometric measures at baseline and follow-up was collected by well-trained nurses using a standard protocol, which would minimise information errors and misclassification bias due to self-reporting or changes in measurement methods, and might enhance comparability of the adiposity measures between the two time points. Furthermore, in older adults, the body fat distribution may change with an increased centralisation of adiposity while total weight remains constant, ${ }^{44}$ indicating that BMI and WC might not change simultaneously, and highlighting the importance of using a combination of BMI and WC to examine the associations of adiposity and mortality comprehensively.

Our study had several limitations. First, the number of all-cause and cause-specific deaths, that is, deaths due to CVD or cancer, was small, which could be due to relatively short follow-up time and might have led to an insufficient statistical power and the subsequent non-significant association. Mega cohorts with millions of subjects are needed for breaking down by several variables together (such as sex, age group, health status and adiposity at baseline, and weight loss/gain intention and treatment). Second, as all our participants were aged 50 years or above at baseline, generalisation of the results to younger populations might be limited. Moreover, women were oversampled in this study, as other population-based elderly cohorts. However, we adjusted for sex in data analysis to minimise its potential confounding effect. In addition, within sex and age group, the participants had similar prevalence of chronic diseases to nationally representative samples of the urban Chinese. ${ }^{17}$ Thus, the unbalanced sex ratio might not be a major concern in the current study. Third, as very few participants $(n=415)$ reported intentional weight loss, we could not further analyse the association of intentional weight loss and mortality in our study. Intentional weight loss in older Chinese people was uncommon. Although obesity is traditionally a symbol of wealth and fortune, the increased awareness of the harms of obesity and related problems such as hypertension, dyslipidaemia, diabetes would lead to increasing weight loss intention. Future studies must collect such information and analyse intentional and unintentional weight loss separately. Fourth, due to improving survival from most chronic diseases since the past few decades globally, ${ }^{45}$ studies focusing on mortality only might be increasingly problematic due to the misclassification of the study outcomes. For example, some survivors of CVD or cancer may be misclassified as 'non-disease' and be used as the reference group, which would bias the relative risks towards the null. Finally, residual confounding due to unadjusted, unmeasured or poorly measured variables, such as self-reported physical activity and smoking, and unmeasured height at the first follow-up examination, could not be ruled out, and hence the observed associations should not be interpreted causally.

In conclusion, BMI gain in those with obesity was associated with a higher risk of all-cause and cancer mortality, suggesting keeping a stable weight at old age could be more beneficial. However, unintentional BMI/WC loss was associated with substantially higher risks of all-cause and CVD mortality, especially in those underweight. As attributable fraction in the exposed group can be calculated by using relative risk minus one, divided by the relative risk, based on an HR of 2.0 for all-cause mortality, an attributable fraction of $50 \%$ can be obtained, which means that of all deaths in older people with both baseline underweight and unintentional weight loss within a few years, half can be attributable to weight loss probably caused by underlying health conditions. ${ }^{46}$ Such results indicate that older and underweight people should be monitored frequently for unintentional weight loss as an early warning for timely clinical investigations and interventions. Moreover, interventions for older people, such as nutrition education and promotion of physical activity, before the fluctuations in weight become evident are important. Future cohort studies should refocus from reverse causality to examine the factors for unintentional weight loss and the adverse health outcomes in the old and thin.

Contributors YYH, LX, THL, GNT, WSZ, FZ, YLJ, CQJ and KKC have made substantial contributions to conception and design, acquisition of funding, data and interpretation of data; YYH, LX, CQJ and THL analysed the data, YYH, LX, CQJ, THL, GNT and KKC drafted the article, LX, CQJ, THL and KKC revised it critically for important intellectual content. All authors read and approved the final manuscript.

Funding This work was supported by the Natural Science Foundation of Guangdong (grant number 2018A030313140), the University of Hong Kong Foundation for Educational Development and Research (grant number SN/1f/ HKUF-DC;C20400.28505200), the Health Medical Research Fund (grant number HMRF/13143241) in Hong Kong; the Guangzhou Science and Technology Bureau, Guangzhou, China (grant number 201704030132).

Competing interests None declared.

Patient consent for publication All participants gave written, informed consent before participation.

Ethics approval The Guangzhou Medical Ethics Committee of the Chinese Medical Association approved the study.

Provenance and peer review Not commissioned; externally peer reviewed.

Data availability statement No additional data are available.

Supplemental material This content has been supplied by the author(s). It has not been vetted by BMJ Publishing Group Limited (BMJ) and may not have been peer-reviewed. Any opinions or recommendations discussed are solely those of the author(s) and are not endorsed by BMJ. BMJ disclaims all liability and responsibility arising from any reliance placed on the content. Where the content includes any translated material, BMJ does not warrant the accuracy and reliability of the translations (including but not limited to local regulations, clinical guidelines, terminology, drug names and drug dosages), and is not responsible for any error and/or omissions arising from translation and adaptation or otherwise.

Open access This is an open access article distributed in accordance with the Creative Commons Attribution Non Commercial (CC BY-NC 4.0) license, which permits others to distribute, remix, adapt, build upon this work non-commercially, and license their derivative works on different terms, provided the original work is 
properly cited, appropriate credit is given, any changes made indicated, and the use is non-commercial. See: http://creativecommons.org/licenses/by-nc/4.0/.

\section{ORCID iD}

Lin Xu http://orcid.org/0000-0002-0537-922X

\section{REFERENCES}

1 NCD Risk Factor Collaboration (NCD-RisC). Worldwide trends in body-mass index, underweight, overweight, and obesity from 1975 to 2016: a pooled analysis of 2416 population-based measurement studies in 128.9 million children, adolescents, and adults. Lancet 2017;390:2627-42.

2 Xu L, Lam TH. Stage of obesity epidemic model: learning from tobacco control and advocacy for a framework convention on obesity control. J Diabetes 2018;10:564-71.

3 Flegal KM, Kit BK, Orpana H, et al. Association of all-cause mortality with overweight and obesity using standard body mass index categories: a systematic review and meta-analysis. JAMA 2013;309:71-82.

4 Di Angelantonio E, Bhupathiraju S, Wormser D, et al. Body-Mass index and all-cause mortality: individual-participant-data metaanalysis of 239 prospective studies in four continents. Lancet 2016;388:776-86.

5 Cheng FW, Gao X, Jensen GL. Weight change and all-cause mortality in older adults: a meta-analysis. J Nutr Gerontol Geriatr 2015;34:343-68.

6 Karahalios A, English DR, Simpson JA. Change in body size and mortality: a systematic review and meta-analysis. Int J Epidemiol 2017:46:526-46

7 Ho SC, Woo J, Sham A. Risk factor change in older persons, a perspective from Hong Kong: weight change and mortality. $J$ Gerontol 1994;49:M269-72.

8 Yaari S, Goldbourt U. Voluntary and involuntary weight loss: associations with long term mortality in 9,228 middle-aged and elderly men. Am J Epidemiol 1998;148:546-55.

9 Nanri A, Mizoue T, Takahashi Y, et al. Weight change and all-cause, cancer and cardiovascular disease mortality in Japanese men and women: the Japan public health Center-Based prospective study. Int $J$ Obes 2010;34:348-56.

$10 \mathrm{He}$ Y, Lam TH, Jiang B, et al. Changes in BMI before and during economic development and subsequent risk of cardiovascular disease and total mortality: a 35-year follow-up study in China. Diabetes Care 2014;37:2540-7.

11 Mousavi SV, Mohebi R, Mozaffary A, et al. Changes in body mass index, waist and hip circumferences, waist to hip ratio and risk of all-cause mortality in men. Eur J Clin Nutr 2015;69:927-32.

12 Lam TH, Xu L. Commentary: fundamental limitations of metaanalysis of cohort studies, epidemiological challenges and the stage of obesity epidemic. Int J Epidemiol 2017;46:547-8.

13 Myrskylä M, Chang VW. Weight change, initial BMI, and mortality among middle- and older-aged adults. Epidemiology 2009;20:840-8.

14 Bamia C, Halkjaer J, Lagiou P, et al. Weight change in later life and risk of death amongst the elderly: the European prospective investigation into cancer and Nutrition-Elderly network on ageing and health study. J Intern Med 2010;268:133-44.

15 Wang YF, Tang Z, Guo J, et al. Bmi and BMI changes to all-cause mortality among the elderly in Beijing: a 20 -year cohort study. Biomed Environ Sci 2017;30:79-87.

$16 \mathrm{Xi} \mathrm{B}$, Liang Y, He T, et al. Secular trends in the prevalence of general and abdominal obesity among Chinese adults, 1993-2009. Obes Rev 2012:13:287-96.

17 Jiang C, Thomas GN, Lam TH, et al. Cohort profile: the Guangzhou Biobank cohort study, a Guangzhou-Hong Kong-Birmingham collaboration. Int J Epidemiol 2006;35:844-52.

$18 \mathrm{Xu}$ L, Jiang CQ, Lam TH, et al. Sleep duration and memory in the elderly Chinese: longitudinal analysis of the Guangzhou Biobank cohort study. Sleep 2014;37:1737-44.

19 Xu L, Jiang CQ, Schooling CM, et al. Liver enzymes and incident diabetes in China: a prospective analysis of 10764 participants in the Guangzhou Biobank Cohort Study. J Epidemiol Community Health 2015;69:1040-4.

$20 \mathrm{Xu} \mathrm{L}$, Lam TH, Jiang CQ, et al. Adiposity and incident diabetes within 4 years of follow-up: the Guangzhou Biobank cohort study. Diabet Med 2017;34:1400-6.

21 Consultation WHOE, WHO Expert Consultation. Appropriate bodymass index for Asian populations and its implications for policy and intervention strategies. Lancet 2004;363:157-63.
22 Alberti KGMM, Eckel RH, Grundy SM, et al. Harmonizing the metabolic syndrome: a joint interim statement of the International diabetes Federation Task force on epidemiology and prevention; National heart, lung, and blood Institute; American heart association; world heart Federation; international atherosclerosis Society; and international association for the study of obesity. Circulation 2009;120:1640-5.

23 Lee JS, Visser M, Tylavsky FA, et al. Weight loss and regain and effects on body composition: the health, aging, and body composition study. J Gerontol A Biol Sci Med Sci 2010;65:78-83.

24 Pan X-F, Yuan J-M, Koh W-P, et al. Weight change in relation to mortality in middle-aged and elderly Chinese: the Singapore Chinese Health study. Int J Obes 2019;43:1590-1600.

25 Dahl AK, Fauth EB, Ernsth-Bravell M, et al. Body mass index, change in body mass index, and survival in old and very old persons. J Am Geriatr Soc 2013;61:512-8.

26 Wang T, Jiang CQ, Xu L, et al. White blood cell count and all-cause and cause-specific mortality in the Guangzhou Biobank cohort study. BMC Public Health 2018;18:1232.

$27 \mathrm{Xu} \mathrm{L}$, Lam TH, Jiang CQ, et al. Egg consumption and the risk of cardiovascular disease and all-cause mortality: Guangzhou Biobank cohort study and meta-analyses. Eur J Nutr 2019;58:785-96.

28 Deng HB, Macfarlane DJ, Thomas GN, et al. Reliability and validity of the IPAQ-Chinese: the Guangzhou Biobank cohort study. Med Sci Sports Exerc 2008;40:303-7.

29 Xu L, Lam TH, Jiang CQ, et al. Changes in adiposity in an older Chinese population in rapid economic transition. Obesity 2016;24:2217-23.

30 Andersen PK, Abildstrom SZ, Rosthøj S. Competing risks as a multistate model. Stat Methods Med Res 2002;11:203-15.

31 Satagopan JM, Ben-Porat L, Berwick M, et al. A note on competing risks in survival data analysis. Br J Cancer 2004;91:1229-35.

32 Miller SL, Wolfe RR. The danger of weight loss in the elderly. J Nutr Health Aging 2008;12:487-91.

33 Landi F, Cruz-Jentoft AJ, Liperoti R, et al. Sarcopenia and mortality risk in frail older persons aged 80 years and older: results from ilSIRENTE study. Age Ageing 2013;42:203-9.

34 Somes GW, Kritchevsky SB, Shorr RI, et al. Body mass index, weight change, and death in older adults: the systolic hypertension in the elderly program. Am J Epidemiol 2002;156:132-8.

$35 \mathrm{Kim}$ Y-H, Kim SM, Han K-do, et al. Change in weight and body mass index associated with all-cause mortality in Korea: a nationwide longitudinal study. J Clin Endocrinol Metab 2017;102:4041-50.

36 Sun Q, Townsend MK, Okereke Ol, et al. Adiposity and weight change in mid-life in relation to healthy survival after age 70 in women: prospective cohort study. BMJ 2009;339:b3796.

37 Nguyen ND, Center JR, Eisman JA, et al. Bone loss, weight loss, and weight fluctuation predict mortality risk in elderly men and women. $J$ Bone Miner Res 2007;22:1147-54.

38 Arnold AM, Newman AB, Cushman M, et al. Body weight dynamics and their association with physical function and mortality in older adults: the cardiovascular health study. J Gerontol A Biol Sci Med Sci 2010;65:63-70.

39 Lee CG, Boyko EJ, Nielson CM, et al. Mortality risk in older men associated with changes in weight, lean mass, and fat mass. J Am Geriatr Soc 2011;59:233-40.

40 Karahalios A, Simpson JA, Baglietto L, et al. Change in body size and mortality: results from the Melbourne Collaborative cohort study. PLoS One 2014;9:e99672.

41 Mulligan AA, Lentjes MAH, Luben RN, et al. Weight change and 15 year mortality: results from the European Prospective Investigation into Cancer in Norfolk (EPIC-Norfolk) cohort study. Eur J Epidemiol 2018;33:37-53.

42 Thomas EL, Frost G, Taylor-Robinson SD, et al. Excess body fat in obese and normal-weight subjects. Nutr Res Rev 2012;25:150-61.

43 de Heredia FP, Gómez-Martínez S, Marcos A, Obesity MA. Obesity, inflammation and the immune system. Proc Nutr Soc 2012;71:332-8.

44 Sun Y, Liu B, Snetselaar LG, et al. Association of normal-weight central obesity with all-cause and cause-specific mortality among postmenopausal women. JAMA Netw Open 2019;2:e197337.

45 DALYs GBD, Collaborators H. Global, GBD 2017 DALYs and HALE Collaborators. Global, regional, and national disability-adjusted life-years (DALYs) for 359 diseases and injuries and healthy life expectancy (HALE) for 195 countries and territories, 1990-2017: a systematic analysis for the global burden of disease study 2017 . Lancet 2018;392:1859-922.

46 Lam TH. Absolute risk of tobacco deaths: one in two smokers will be killed by smoking. Arch Intern Med 2012;172:845-6. 